\title{
Orbits Connectivity and the Asymptotic Behavior at Iterated Functions with Lyapunov Stability
}

\author{
Charles Roberto Telles \\ Secretary of State for Education and Sport of Paraná. Curitiba, Brazil \\ E-mail: charlestelles@seed.pr.gov.br
}

\begin{abstract}
By defining a constant probabilistic orbit of $f(x)$ and $g(x)$ iterated functions, the stability dynamics of these functions in possible interactions through connectivity provides the formation of a dynamic fixed point $S_{n}$ as a metric space between both iterated functions. The presence of a dynamic fixed point identifies qualitatively phases of iteration time lengths and interaction orbits of the event. Qualitative results show that the greater the average distance from one of the functions to the fixed point of the other (all possible solutions), the higher the iteration expression on time (false asymptotic effect) of one of the functions and in the opposite hand, the lower the average distance, the higher orbit's interactions proximity between iterated functions (stability). This feature reveals asymptotic (welldefined) behavior between functions $f$ and $g$ within a well-defined Lyapunov stability.
\end{abstract}

Keywords: iterated function; asymptotic analysis; discretization; stability theory; metric space; qualitative theory of differential equations.

\section{Introduction}

Although asymptotic iterated functions can be often considered empirically invisible in their nature of proportionality [1], the expression frequency of iterations with which these functions occur partially on time in their formulations affecting the physical nature of a phenomenon are subject to observation and discrimination to the extent that they can be observed knowing the metric of spaces that constitute all the stages of a given event in terms of its iterated functions be a product of interactions $[1,2]$. However, as the metric space of these iterated functions oscillates in their expressions and can increase distances from each other in terms of possible interactions (monotone functions) [2], it is possible to describe the event as presenting a dynamic asymptotic imbalance between functions in terms of expression in time or space for all finite solutions directed to a fixed point as noted by Lundberg (1963) [2] but, as defined in this research, a dynamic fixed point, resembling Williamson's concept (1991) [3]. In this sense, for identifying at Lyapunov stability an asymptotic behavior, it is necessary to describe an event considering its escaping and approximation orbits in order to make visible, mathematically, the asymptotic formation feature within the apparent stable dynamics. However, in this research it will be demonstrated that the apparent asymptotic instability observed in nature function expression in connectivity is in true a false asymptotic effect. This feature occurs as far as the time confers to a given function and its interaction, a quantified empirical result that will express in higher proportion than other iterated functions and its interactions that don't have enough time lengths to express. The camouflage effect resides in the sense that the asymptotic definition in terms of empirical observation does not match with true function equation, being the effect of time lengths a tool in which empirical results can present massive quantified results of a function that can even be not asymptotic at all.

It is defined as a dynamic fixed point in this research the formation by iteration and interaction between distinct iterated functions, of a metric space in which one of the functions that are defined partially by a fixed point assume other distances between two points of two distinct functions, alternating the position in the metric space between one function and the other.

This connective metric space in turn generates a kind of instability between the fixed point metric position that can be defined in a specific order of possible orbits of interactions in the system (solutions 
proximity) [4] and at the same time a region of space in which the iteration frequencies of the iterated functions reach their maximum degree of expression presenting higher distance between two points of both functions (escaping solutions) $[5,6,7]$. Thus, Banach's fixed-point theorem objectively illustrates the distribution of iterated functions over a single fixed point in a complete metric space, considering for it the dynamic fixed point concept and reflecting the definition of one of the iterated functions partial attraction to a fixed point for each interaction, but in a connectivity metric space [8].

To analyze the possibility of the formulations described in this introduction, the methodology section will address a case of iterated functions that will be described at the end, in the results section, in graphical terms of the expression that evidences the presence of regions of the metric space with the largest amount of orbits between functions (interaction) and expression frequency on time (iteration itself).

\section{Methodology}

\subsection{Connectivity and iterated functions.}

Theorem 1. Consider that two functions $f$ and $g$ iterated in connectivity and a complete space metric $x_{n+1}$, present in common an interaction with a third domain $y$ defined symmetrically as a mirror of $f$ and $g$ maps. These two iterated functions in terms of their physical nature have defined probabilistic temporal / spatial behavior parameters. The time of occurrence $t_{n+1}$ for each event representing each function iterated in connectivity is constant at a expression frequency of time as $t_{n+1}+t_{n+1}$ and probabilistic distribution $P_{n}$, constant. The interaction of these two functions in a given region of the metric space $x_{n+1}$ generates, assuming that the quantitative properties of the event remain with partial and asymmetric numerical transformations to their original form $(\partial)$, for each interaction such as,

$$
\frac{\partial x_{n+1}}{\partial g}(f, g)=0 \therefore x_{n+1}(f, g)=f(f)
$$

and vice versa for $f(g)$, a solution to a fixed point at $y$ that defines itself as a random variable $\partial x_{n+1}=S_{1}, \ldots, S_{n}$ (dynamic fixed point) for only one of the functions $f(x)$ or $g(x)$ at each interaction. The distance between two points in each iterated function at $y$ occurs in a general and maximum expansion as,

$$
d(f(x), g(x))=d\left((g(x)=f x+g x) \leq \sum_{S_{n}+1}^{n} S_{n}\right.
$$

and vice versa. Where $x_{n+1}$ can be represented by a connectivity in which the global distance between two points of function domains assume distances equal to,

$$
d\left(f\left(g\left(S_{n}\right)\right), g(x)\right) \leq d\left(\sum_{S_{n}+1}^{n} S_{n}, f\left(g\left(S_{n}\right)\right),\right.
$$

and vice versa. The convergence of one of the functions when attracted to a fixed point is defined as $\lim _{n \rightarrow \infty}\left(S_{n+1}-S_{n}\right)=0$, where $S_{n}+1$ is a metric space in which $g(x)$ or in turn $f(x)$ are not defined at the fixed point mutually as indicated in number notation (4).

$$
\begin{aligned}
& f: f\left(g\left(S_{n}\right)\right) \rightarrow S_{n+1} \\
& g: g\left(f\left(S_{n}\right)\right) \rightarrow S_{n-1}
\end{aligned}
$$

This means that to the extent that one of the iterated functions is dynamically defined by fixed points, there is the formation of fixed positions in space and time with which the distance between two points of both sets for each interaction assumes Lyapunov stability [9] with a bounded mapping condition where for all vectors, the solutions will always be less than or equal to the sum of all dynamic fixed points $\left(v \leq \sum_{S_{n}+1}^{n} S_{n}\right)$. When observing the connectivity of the iterated event, this characteristic (connectivity) reveals that the maximum dynamic fixed points are formed as Cartesian product of the iterated functions generating a defined phase space (locally stable or unstable according to fixpoint position in the phenomenon) by the sequence (orbits) of interaction between functions and the average distances generated between the points of each function for each sequence of interactions (orbits). This behavior of the event also allows us to observe, as will be described in the results section, that the distance between two points of each domain, in the dynamics of maximum possible interactions and synchronously, assume 
an asymptotic instability that accompanies certain empirical expressions, in which one of Iterated functions in connectivity may not be visible (discretized) due to an asymptotic camouflage effect.

This event can best be described as an orderly sequence of iterations $x_{n+1}+t_{n+1}=1$ with parameters determined in $S_{n}+1, P_{n}$ e $D$ in the relationship that is defined only from $S_{1}, \ldots, S_{n}$, generating a composition like $(f \circ g)\left(S_{n}\right)$ as $f\left(S_{n}+1\right)=\left\{f\left(S_{n}\right) \mid S_{n} \in S_{n}+1\right\}$.

A linear prediction can be obtained if for certain frequencies defined as Markovian the functions $S_{n}+1$ are infinitely discretized in the available proportion of $S_{n}$, revealing the flow of process iterations as $\int_{f(x)}^{g(x)}\left(f^{m} \circ g^{n}\right)=\left(S_{n}^{m+n}\right)$ in its various position dynamics at a single fixed point and for the formation of other dynamic fixed points.

To visualize the interaction of iterated functions and the formation of connectivity, the following sets are defined as an example in figure 1.

Lemma 1. It is possible to observe that the fixed points $S_{n}$ are generated from the demand $S_{n}+$ 1. Thus, event discretization $S_{n}+1$ occurs as a sequence is organized from the availability of $S_{n}$, which these fixed points in turn assume a function defined as $(f \circ g)(x)$. Therefore, for both functions $f$ and $g$, the iterations of each function remain as an image of interactions occurring at a given moment from $n$ fixed points $S_{1}, \ldots, S_{n}$ randomly or not, generated as $f:\left(S_{n}+1\right)+\left(S_{n}+1\right) \rightarrow S_{n} \therefore f \times g$, where the metric space of connectivity between two points of each iterated function is defined as $d\left(f\left(g\left(S_{n}\right)\right), g(x)\right) \leq d\left(\sum_{S_{n}+1}^{n} S_{n}, f\left(g\left(S_{n}\right)\right)(3)\right.$, and vice versa.

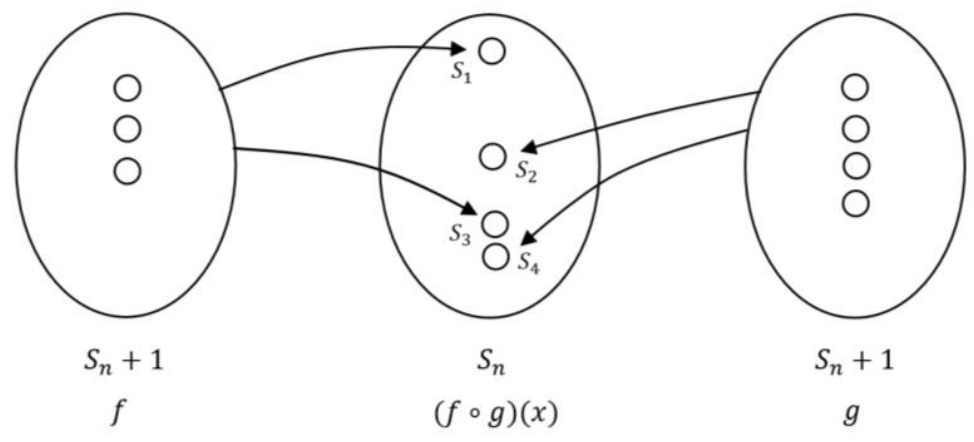

Figure 1. Formation of dynamic fixed points by connectivity $(f \circ g)(x)$.

Proof of theorem 1 and lemma 1. The equation that describes the behavior of figure 1 for each iterated function in terms of space and time can be described as:

$$
S_{n}+1=\left(x_{n+1}+t_{n+1}\right) P_{n}
$$

Since all variables of each function have defined values, then the interaction orbits have homogeneous characteristics as found in Lyapunov's stability,

Where,

$$
\left(S_{n}+1\right) n=\left(x_{n+1}+t_{n+1}\right) P_{n}
$$

Therefore,

$$
L=(f \circ g)(x) \therefore(f \circ g)\left(S_{n}\right)
$$

$$
\sum_{S_{n}+1}^{n} S_{n}=S_{1}+S_{2}+S_{3}+\cdots+S_{n}
$$

Hence, connectivity can be defined by the equations,

Q.E.D.

$$
f(x)=\sum_{S_{n}+1}^{n} S_{n}-g(x) \text { or } \sum_{S_{n}+1}^{n} S_{n}=f(x)+g(x) \text { or } g(x)=-f(x)+\sum_{S_{n}+1}^{n} S_{n}
$$

Following this definition, all numerical solutions directly reflect the maximum number of locally stable and unstable maps [9], sequence of interactions and expression frequency of iterated functions on time, and the visible expression of the event in empirical terms of analysis (considering that the quantized nature of the event is known at certain margins of variation or deterministic definition in a sensorial dimension of events). 
As the event unfolds, the metric space between $f(x)$ and $g(x)$ in connectivity to $S_{n}$, for all dynamic fixed points can be set globally per interaction as:

$$
d\left(f\left(S_{n}+1\right), g\left(S_{n}+1\right)\right)=\left(S_{n}+1\right)-\sum_{S_{n}+1}^{n} S_{n} d\left(S_{n}+1, S_{n}+1\right)
$$

And locally per iteration, roughly defined as,

$$
d\left(f\left(S_{n}+1\right), g\left(S_{n}+1\right)\right)=\left(S_{n}+1\right)-\sum_{S_{n}+1}^{n} S_{n} d\left(f\left(g\left(S_{n}\right)\right), g(x)\right) \text { or }\left(g\left(f\left(S_{n}\right)\right), f(x)\right)
$$

Both the function $f(x)$ as $g(x)$ are false asymptotic to each other symmetrically as far as one function or the other is increasing only in time lengths.

$$
x \rightarrow \infty \text { for } f \text { or } g \therefore f(x) \sim g(x) \text { or } g(x) \sim f(x)
$$

Given the expressions of $f(x)$ and $g(x)$, you can define that iterations are defined through $S_{n}$ in order that $\left(f^{m} \circ g^{n}\right)=\left(S_{n}^{m+n}\right)$. However, it should be noted that in the expression $S_{n}+1=f(x)+$ $g(x)$, it can be converted into $\int_{f(x)}^{g(x)}\left(f^{m} \circ g^{n}\right)=\left(S_{n}^{m+n}\right)$, where the sum of existing functions can reach as many iterations as possible in the bounded event, however, many of the iterations are identical and it is not possible to observe event variations in an empirical sense, which in this article is treated as the invisible effect of the numerical intervals in a true asymptotic equation (dynamic fixed points and the empirical expression of iterated functions). Thus, although not visible, both functions clearly differ in terms of empirical properties of the iteration and their expressions within a context, for example, probabilistic distribution for linear / nonlinear events which directly affects the ability to discretize the event, quantify it and have control over the sequence of interactions and frequency of iterations over time. Therefore, since functions assume a bounded characteristic, it is really a parameter on which asymptotic effects can be neglected while causing the most complex events or in the other direction, it in fact can't even exist. So when in $f(x) \sim g(x)$, it is obtained the smallest metric space of iterations between two points of each set in $f(x)$, consequently $g(x)$ assumes greater distance from the fixed point of $f(x)$ making it possible higher time lengths occur for the events of $g(x)$ because of their relative distance (average) in the metric space of connectivity between two points between sets. And vice versa.

For all cases defined by theorem 1 and lemma 1, we have a graphical area projection in which $\int_{f(x)}^{g(x)}\left(f^{m} \circ g^{n}\right)=\left(S_{n}^{m+n}\right)$ obtain lower time length of interactions in the locally asymptotically stable region $p$ and higher time length in the locally false asymptotically unstable region $r$ as follows in Figure 2.

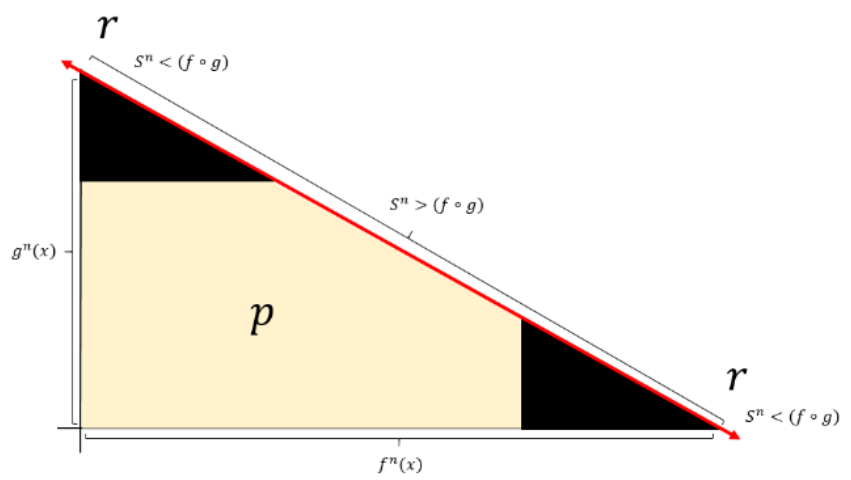

Figure 2. Expression frequency of interactions and iterations on time between iterated functions from a dynamic fixed point in connectivity.

If the number of iterated functions equals $S_{n}$, and exhibiting the same behavior as described in the methodology section as a Cartesian interaction product, the false asymptotic effect closes as the distances between dynamic fixed points for each of the interactions in connectivity are as short as possible (all possible solutions will converge in the neighborhood of $f\left(g\left(S_{n}\right)\right)$ or $g\left(f\left(S_{n}\right)\right)$ ). If the number of functions exceeds $S_{n}$, queue theory is the best description of the event because it directly reflects the theoretical foundations of this knowledge. 


\section{Results}

\subsection{Dynamical fixed points and connectivity metrics.}

Consider two iterated functions in connectivity $(f \circ g)\left(S_{n}\right)$ which in turn defines the dynamics of the fixed point $S_{n}$ according to methodology section. In this sense, the metric space assumes behavior like $d(f(x), g(x))=d\left((g(x)=f(x)+g(x)) \leq \sum_{S_{n}+1}^{n} S_{n}\right.$ and vice versa.

Table 1. Events $f\left(g\left(S_{n}\right)\right)$ and $g\left(f\left(S_{n}\right)\right)$ in connectivity at $S_{n}$.

\begin{tabular}{|c|c|c|c|c|}
\hline \multicolumn{5}{|c|}{ Dynamic fixed point $\left(\sum_{S_{n}+1}^{n} S_{n}\right)$} \\
\hline$f\left(g\left(S_{n}\right)\right)$ & \multicolumn{4}{|c|}{$g\left(f\left(S_{n}\right)\right)$} \\
\hline$g\left(f\left(S_{n}\right)\right)$ & $f\left(g\left(S_{n}\right)\right)$ & \multicolumn{3}{|c|}{$g\left(f\left(S_{n}\right)\right)$} \\
\hline \multicolumn{2}{|c|}{$g\left(f\left(S_{n}\right)\right)$} & $f\left(g\left(S_{n}\right)\right)$ & \multicolumn{2}{|c|}{$g\left(f\left(S_{n}\right)\right)$} \\
\hline \multicolumn{3}{|c|}{$g\left(f\left(S_{n}\right)\right)$} & $f\left(g\left(S_{n}\right)\right)$ & $g\left(f\left(S_{n}\right)\right)$ \\
\hline \multicolumn{4}{|c|}{$g\left(f\left(S_{n}\right)\right)$} & $f\left(g\left(S_{n}\right)\right)$ \\
\hline
\end{tabular}

First and last lines represent the locally asymptotically stable iterations and interactions events, while second to forth lines represent the asymptotically unstable iterations and interactions events.

Or the opposite direction of $f\left(g\left(S_{n}\right)\right)$,

Table 1. Events $g\left(f\left(S_{n}\right)\right)$ and $f\left(g\left(S_{n}\right)\right)$ in connectivity at $S_{n}$.

Dynamic fixed point $\left(\sum_{S_{n}+1}^{n} S_{n}\right)$

\begin{tabular}{|c|c|c|c|c|}
\hline$g\left(f\left(S_{n}\right)\right)$ & \multicolumn{3}{c|}{$f\left(g\left(S_{n}\right)\right)$} \\
\hline$f\left(g\left(S_{n}\right)\right)$ & $g\left(f\left(S_{n}\right)\right)$ & \multicolumn{2}{c|}{$f\left(g\left(S_{n}\right)\right)$} \\
\hline$f\left(g\left(S_{n}\right)\right)$ & $g\left(f\left(S_{n}\right)\right)$ & \multicolumn{2}{c|}{$f\left(g\left(S_{n}\right)\right)$} \\
\hline \multicolumn{2}{|c|}{$f\left(g\left(S_{n}\right)\right)$} & $g\left(f\left(S_{n}\right)\right)$ & $f\left(g\left(S_{n}\right)\right)$ \\
\hline \multicolumn{2}{|c|}{$f\left(g\left(S_{n}\right)\right)$} & & $g\left(f\left(S_{n}\right)\right)$ \\
\hline
\end{tabular}

First and last lines represent the locally asymptotically stable iterations and interactions events, while second to forth lines represent the asymptotically unstable iterations and interactions events.

In Tables 1 and 2, it is possible to observe the Cartesian alternating sequence of functions that reaches a maximum of possible combinations.

Using the formula (9) of the methodology section and the position in the metric space defined in (3), the events in tables 1 and 2 can be described as:

$$
\begin{gathered}
f: f\left(g\left(S_{n}\right)\right) \rightarrow S_{n+1} \\
g: g\left(f\left(S_{n}\right)\right) \rightarrow S_{n-1} \\
f(x)=\sum_{S_{n}+1}^{n} S_{n}-g(x) \\
\text { If } f\left(g\left(S_{n}\right)\right)=1, \text { then, } 1=(5+1)-g\left(f\left(S_{n}\right)\right) \\
+\left(-g\left(f\left(S_{n}\right)\right)=1-6\right) \\
g\left(f\left(S_{n}\right)\right)=5
\end{gathered}
$$

By following this path, the average distances can be briefly obtained as:

$$
\begin{aligned}
& f\left(g\left(S_{n}\right)\right)=1 \quad \therefore g\left(f\left(S_{n}\right)\right)=5 \\
& f\left(g\left(S_{n}\right)\right)=2 \quad \therefore g\left(f\left(S_{n}\right)\right)=4 \\
& f\left(g\left(S_{n}\right)\right)=3 \quad \therefore g\left(f\left(S_{n}\right)\right)=3 \\
& f\left(g\left(S_{n}\right)\right)=4 \quad \therefore g\left(f\left(S_{n}\right)\right)=2 \\
& f\left(g\left(S_{n}\right)\right)=5 \quad \therefore g\left(f\left(S_{n}\right)\right)=1
\end{aligned}
$$

Resulting in a chart with Cartesian coordinates of the equation $\int_{f(x)}^{g(x)}\left(f^{m} \circ g^{n}\right)=\left(S_{n}^{m+n}\right)$ and the values found in solutions (14) as in figure 3. 


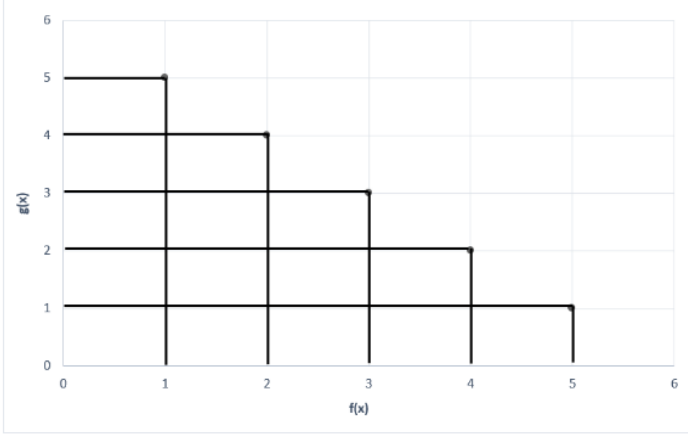

Figure 3. Cartesian iterated function distribution of $f\left(g\left(S_{n}\right)\right)$ and $g\left(f\left(S_{n}\right)\right)$ at $\sum_{S_{n}+1}^{n} S_{n}$.

The expression frequency of iteration functions on time in a Cartesian form can be defined as:

$$
\int_{f(x)}^{g(x)}\left(f^{m} \circ g^{n}\right)=\left(S_{n}^{m+n}\right)
$$

Where in the given example, the exponent indicates the number of distinct positions of each function within the metric space, or in other words, the Lyapunov stability interaction dynamics and iterations of the functions,

$$
\int_{f(x)}^{g(x)}\left(f^{5} \circ g^{5}\right)=\left(S_{n}^{10}\right) \text { or } S_{1}+S_{2}, \ldots,+S_{10}
$$

Where both iteration frequencies for both functions do not match in either position $S_{n}$ for any kind of connectivity in $f\left(g\left(S_{n}\right)\right)$ or $g\left(f\left(S_{n}\right)\right)$.

And to extract the iteration values in $S_{n}^{10}$, the largest and smallest distances of iterated functions from the dynamic fixed point in $f(x)$ or $g(x)$, can be obtained by viewing the maximum distances between $f(x)$ and $g(x)$ as the smallest number of event interactions (phase I) for either function, depending on your position $S_{n}$, which increases the time length of iterations as a canonical phase space, and the minimum distances as the largest number of event interactions (reducing the time length of each iterated function - phase II). This definition can be seen in figure 4.

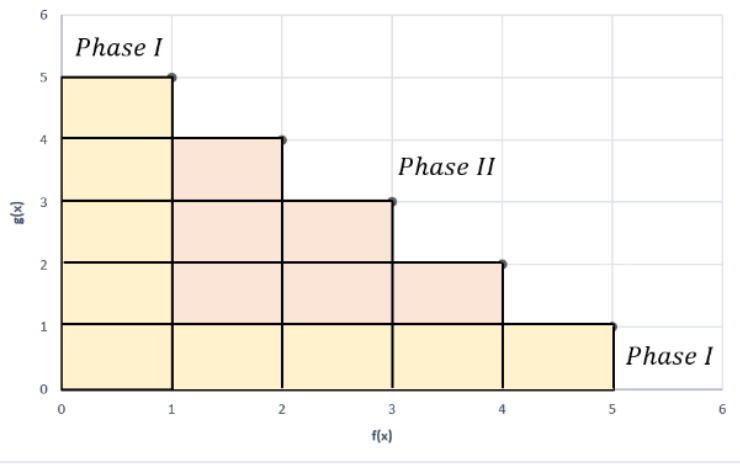

Figure 4. Distribution of functions $f\left(g\left(S_{n}\right)\right)$ and $g\left(f\left(S_{n}\right)\right)$ at $\sum_{S_{n}+1}^{n} S_{n}$ defining two distinct phase regions.

Respectively, Phase I represents high time length regions of iterations in one function (false asymptotically unstable) and Phase II, regions where the time length of iterations have solutions that tend to the fixed points of one function (asymptotically stable). Note that for illustration purposes phase II was colored light orange, but it is not defined only in this colored region.

Analyzing phase spaces in the iterated functions at a dynamic fixed point, the vectors variations that can be obtained by a fixed point dynamics in terms of time and space are quite expressive in possibilities. By illustrating in Figure 5 a sequence of dynamic fixed points that occur according to Table 1, 
it is possible to observe the iterated functions in connectivity and the geometric aspect of the iterations and interactions orbits/frequencies of one of the possible connectivity $f: f\left(g\left(S_{n}\right)\right) \rightarrow S_{n}$.

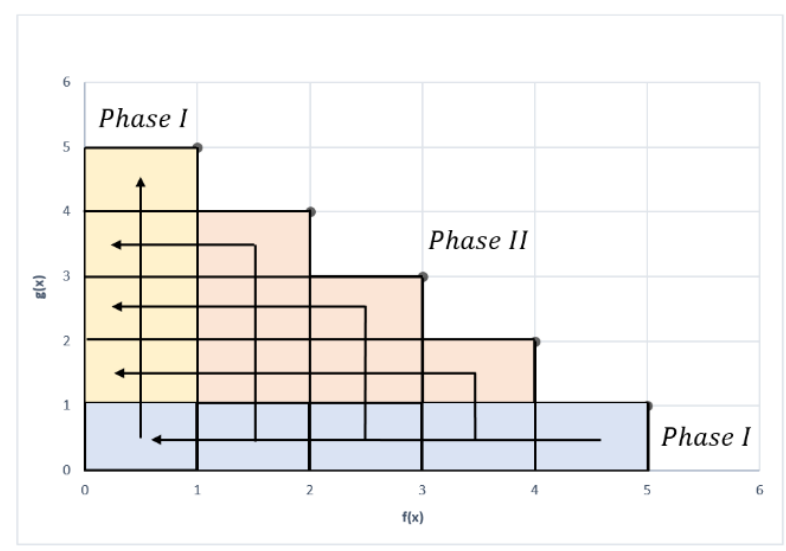

Figure 5. Maximum and minimum distances of functions $f\left(g\left(S_{n}\right)\right)$ and $g\left(f\left(S_{n}\right)\right)$ at $\sum_{S_{n}+1}^{n} S_{n}$, iterated. The vectors at each coordinate of $f(x)$ and $g(x)$ represent the different dynamic fixed point formations $S_{n}$, as well as reflect the points with the most orbits of iterated functions and / or frequencies with which each iterated function expresses itself. In phase II in relation to phase I, the region concentrates most of the iterated function orbits according to the distribution obtained in the solutions (14).

In Figure 5 it is possible to identify a geometric expression of the existing vectors for the given fixed point and interactions distributions of the iterated functions. As exemplified in the image in Figure 5 , it is possible to conceive of an orientation in physical space in which solution vectors can assume various fractal shapes, also exhibiting a behavior of fixed point dynamics as discussed in section 3.2.

\subsection{False Asymptotic Instability at Iterated Functions}

Despite of a false asymptotic effect might exist in an iterated function analysis, this research points out that since time lengths poses the true cause of events, it can be also used as a tool to design new results towards quantitative aspect of physical, chemical or biological properties of reality.

By observing the phase space (figure 6) constituted by the object of this research, it is possible to realize that dynamics between asymptotic true and false events and their possibility of manipulation of these events in terms of their interaction and iteration through time lengths.

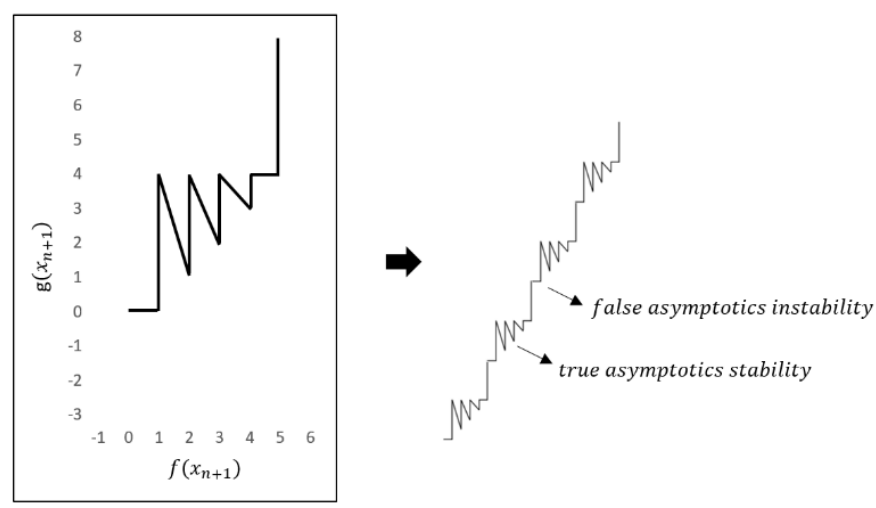

Figure 6. Time series of phases I and II evidencing the distribution of the iterated event between distinct functions for a dynamics of true asymptotic stability and false asymptotic instability. Overall the event presents a stable Lyapunov equilibrium, continuously iterating with the same formation (figure 7.). 


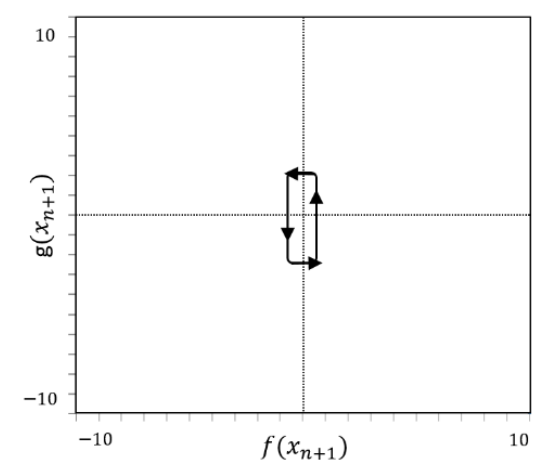

Figure 7. Phase portrait of the example shown in this research showing Lyapunov stability.

This dynamic can be visualized through different possibilities of creating iterated events that alternate in their physical and natural expressions due to interactions in connectivity as shown in Figure 8 (Note that the data was not simulated to construct this image. This image is represented because it satisfies the numerical solution conditions of Figure 3. similar to the Vinograd system, but the regions of false asymptotic instability in $f$ and $g$ would be smaller curves than the image presented). The symmetry region (negative and positive of the graph) represent the iterated functions respectively $f\left(g\left(S_{n}\right)\right)$ and $g\left(f\left(S_{n}\right)\right)$ in $\sum_{S_{n}+1}^{n} S_{n}$ alternately designed by time lengths of both functions at interactions.

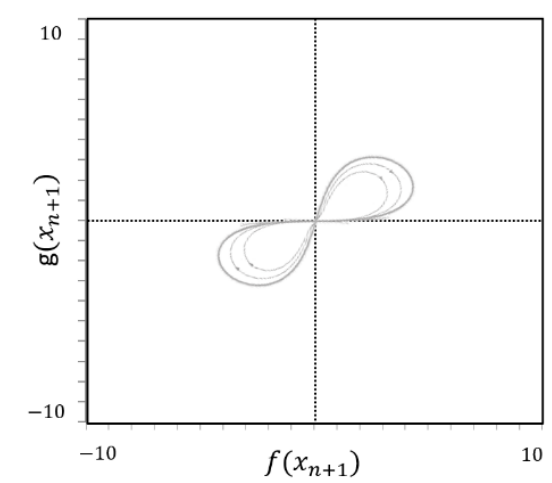

Figure 7. Image from left represents a sequence of interactions and iterations with an idealized spatial form of the event phase space, representing phases I and II of figure 4.

One way of empirically observing the expression of iterated functions in connectivity on time, similar to the descriptions present in this research, would be in figure 8 . In the left image $[10,11]$ a chemical event of wheat is represented in which the time length of iterations and interactions has a false asymptotic instability at the beginning of event $A$ (higher time length of iterations that gives a certain physicochemical property), and asymptotic stability after the initial phase of event B (the loss of physicochemical properties that give rigidity to the material, which is understood to be an asymptotic stability of the event). And in the image at bottom, the closed pine cone biological structure in its fractals and shape [12] can be observed for its asymptotic stability (regular time lengths) at the center of the structure, obtaining maximum interactions and determined time length of iterations, and in the final portion of the structure (tapered) it is possible to observe the false asymptotic instability (higher expression of a given function on time) in which the interactions between the functions become smaller and, consequently, the frequencies on time with which each iterated function expresses become larger.

A

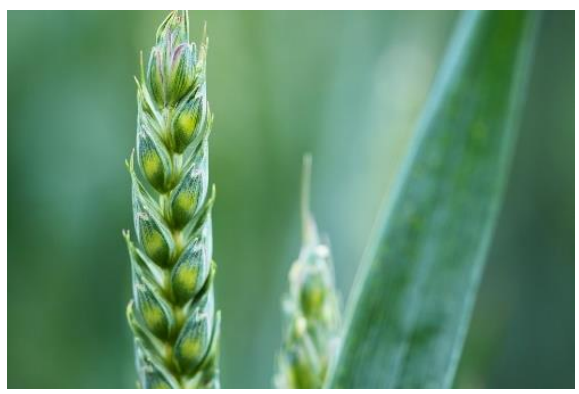

B

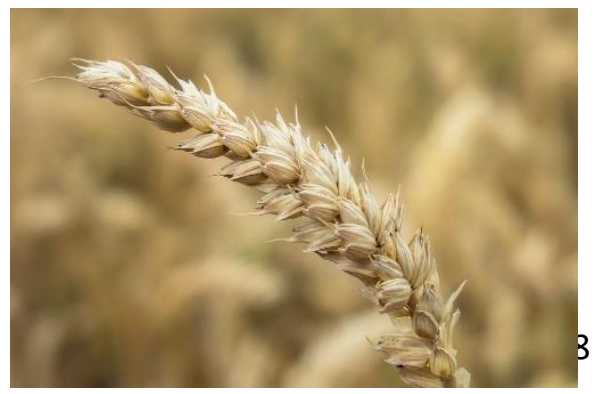




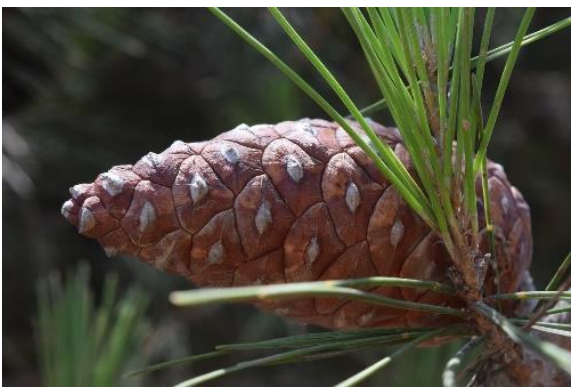

Figure 8. Representation of an event with Lyapunov stability and its stable and unstable localities.

Note that there might be some variations on the fractal and form $f$ closed pine cone as well as rigidity or bendiness of wheat structure due chemical or biological factors such as defective proteins, genes or other environmental conditions. But it does not change the mathematical modeling of describe in this research.

\section{Conclusion}

The greater the average distance between two points between iterated functions in connectivity in the given model, the more often the more distant function will express itself around the fixed point of the one of the iterated function, which characterizes the false asymptotic effect of instability considering the metric space generated at this phase I. Similarly, not only in terms of iteration expression frequency (time length), but iterated functions if observed in terms of their physical nature, are also defined in the possibility that the function has a certain time or space for expression of events iterated and interdependent with other variables. Therefore, in the opposite direction, the smaller the average distance, the lower the expression frequency of iteration on time in both functions proportionally to the fixed point distance due to the metric position that each function occupies in the metric space, which characterizes the stable asymptotic effect.

Globally speaking, the entire event presents a Lyapunov stability due to the constant and periodic orbits defined by specific fixed points, even if dynamically distributed. When taking into account a Lyapunov stability, it is interesting to observe that in the given equilibrium state, the event might present false asymptotic instability and stability as a camouflage effect under the apparent equilibrium of orbits.

In several events involving iterations, such as physics, biology and chemistry phenomena and related knowledge, iteration events are manifestations of repetition in the order or disorder of elements that constitute an iteration $[13,14]$. Although the cause of iterations as well as their behavior are key topics in the search for iterated events, an important aspect raised in this paper reflects on the discretization of iterated events in terms of knowing how behave diverse iterated functions that generates an event, and this observation serves as a tool to understand the flow with which each function infinitely iterates from a fixed point of dynamic type.

If we consider, for example, atmospheric events, in a temperature range between meeting air masses, to be considered as a set A cold mass and a set $B$ hot mass, the interaction between these events necessarily generates a region of instability $C$, which analogously to this study will be the dynamic fixed point that will form at the conjunction of $A$ and $B$, which in turn will also be synchronously affected at event $C$. This example helps to illustrate that the dynamics of continuously iterating atmospheric particles, according to the methodological definitions of this paper, will express behavior in which regions of the space under which it creates a dynamic fixed point have symmetric and asymmetric sets $A$ and $B$ (fixed points dynamics), in turn generating densities of iterations and interactions over space. The so-called minimum and maximum distances of events iterated from a fixed point reveal the regions of space in which particles will have the highest and lowest time length of interaction and iteration, which affects the physical expression effect of particles in physicochemical aspects. The duration of time length of iterations and interactions in different regions of space allows both sets A and B (hot and cold air mass) to express higher or lower time influence on the event with expressions of greater or lesser influence on the linear and nonlinear dynamics of the physical and chemical phenomena effects of the event. 
It is suggested as future research, the refinement of the mathematical formulations described in this research in order to achieve descriptions involving a larger number of iterated functions. Similarly, it is also suggested that the nature of cause and effect iterations from iteration and interaction frequencies and dynamic fixed points serve as sample data about the use of this method to describe behaviors of iterated events in connectivity.

A very important aspect that was not addressed in this article is the time factor as a precursor to iterated events. For natural events, the ergodicity of the variables in interacting can also be regulated by time as an event that is initially conditioned by it and has evolution affected by it. The time factor was not addressed in this article.

Funding: This research received no external funding.

Conflicts of Interest: The author declares no conflict of interest.

\section{References}

[1] de Bruijn NG. An asymptotic problem on iterated functions. In Indagationes Mathematicae (Proceedings) 1979 Jan 1 (Vol. 82, No. 1, pp. 105-110). North-Holland.

[2] Lundberg A. On iterated functions with asymptotic conditions at a fixpoint. Arkiv för Matematik. 1964 Sep 1;5(3):193-206.

[3] Williamson D. Dynamically scaled fixed point arithmetic. In [1991] IEEE Pacific Rim Conference on Communications, Computers and Signal Processing Conference Proceedings 1991 May 9 (pp. 315-318). IEEE.

[4] Natoli C. Fractals as Fixed Points of Iterated Function Systems. University of Chicago. 2012 Aug 26.

[5] Bharucha-Reid AT. Fixed point theorems in probabilistic analysis. Bulletin of the American Mathematical Society. 1976;82(5):641-57.

[6] A. Gupta, R. Jain, and P. Glynn, Probabilistic contraction analysis of iterated random operators, submitted to Annals of Applied Probability, (2018). arXiv preprint arXiv:1804.01195.

[7] Murray RM. A mathematical introduction to robotic manipulation. CRC press; 2017 Dec 14.

[8] Jamison B. Asymptotic behavior of successive iterates of continuous functions under a Markov operator. Journal of mathematical analysis and applications. 1964 Oct 1;9(2):203-14.

[9] Zdun MC. The embedding problem in iteration theory. ESAIM: Proceedings and Surveys. $2014 \mathrm{Nov}$ 1;46:86-97.

[10] Pixabay. Didgeman's image: bended old wheat. Created at July 13, 2019. Retrieved from: https://pixabay.com/photos/wheat-cereals-grain-cornfield-4335863/.

[11] Pixabay. Public domain pictures: green wheat. Created at June 5, 2009. Retrieved from: https://pixabay.com/photos/agriculture-background-cereal-corn-2229/.

[12] Pixabay. Ulleo's image: closed pine cone. Created at June 7, 2016. Retrieved from: https://pixabay.com/photos/pine-tap-pine-cones-nature-closed-1457849/.

[13] Harmati I, Hatwágner MF, Kóczy L. On the existence and uniqueness of fixed points of fuzzy cognitive maps. In Information Processing and Management of Uncertainty in Knowledge Based Systems. Theory and Foundations - 17th International Conference, IPMU 2018, Proceedings. Springer Verlag. 2018. p. 490500. (Communications in Computer and Information Science).

[14] Fromm J. On engineering and emergence. arXiv preprint nlin/0601002. 2006 Jan 3. 